\section{Differential Impact of Sound on Saccades Vergence and Combined Eye Movements: A Multiple Case Study}

\author{
Zoï Kapoula* and Erwann Pain \\ IRIS Laboratory, CNRS FRE2022, University of Paris, F-75506 Paris, France
}

\begin{abstract}
Most of the previous literature regarding eye movements use visual stimuli alone. However, in real life, visual and auditory stimuli occur together frequently. It has been shown that a sound presented just before or simultaneously with the visual target reduces the latency of the saccades. The present study investigates the effect of a sound presented shortly before the LED visual target for three types of eye movements: saccades, vergences and combined eye movements. Fourteen participants ( 6 women, mean age: $22.6+/-0.62$ years) took part in the study. Each type of eye movements was tested in two conditions: one with visual LED targets alone, the other with a sound coming for a buzzer adjacent to the LED, and preceding by $50 \mathrm{~ms}$ the onset of the LED target (auditory-visual target). Eye movements were recorded at $220 \mathrm{~Hz}$ for each eye with the Eyeseecam binocular devicehttps:// www.eyeseetec.de/eyeseecam-sci/). The results confirm that the sound significantly reduces the latency of the saccades. In contrast, for convergence or divergence the sound did not decrease the latency but it did increase the velocity and reduced the duration of such movements significantly. For combined saccade vergence movements particularly leftward, the sound had multiple effects on the saccade component: it decreased the duration of the saccade component and reciprocally increased its velocity but also reduced its latency. Such mixed effects on latency duration and velocity of the saccade component favor the hypothesis that saccade components of combined eye movements are subtended by a co-activation of both, saccade and vergence brainstem generators. The differential benefit from the sound according to the type of eye movements is of theoretical and clinical interest.
\end{abstract}

* ${ }^{*}$ orresponding author: Zoï Kapoula, IRIS laboratory, CNRS FRE2022, University of Paris, F-75506 Paris, France, Tel: +33 0142864039; Email: zoi.kapoula@gmail.com

Citation: Kapoula Z, Pain E (2020) Differential Impact of Sound on Saccades Vergence and Combined Eye Movements: A Multiple Case Study. J Clin Stud Med Case Rep 7: 095.

Received: September 22, 2020; Accepted: September 29, 2020; Published: October 06, 2020

Copyright: ๑ 2020 Kapoula Z, et al. This is an open-access article distributed under the terms of the Creative Commons Attribution License, which permits unrestricted use, distribution, and reproduction in any medium, provided the original author and source are credited.

\author{
Keywords: Saccades; Sound; Vergence; Combined eye \\ movements; Multisensory facilitation
}

\section{Introduction}

Saccades are the rapid movements of the eyes that allow us to abruptly change fixation. Their amplitude ranges from $0-30$ degrees, while the majority of saccades we make in everyday life have amplitudes below 10 degrees. Saccades are the fastest eye movements. Their duration ranges from 20 to $100 \mathrm{~ms}$ depending on their amplitude [1]. Vergence eye movements serve to change the vergence angle of the optic axes so that the fovea of each eye is aligned on a target located at a given depth from the observer. Contrary to saccades, in which the eyes turn in the same direction (conjugate movements), during vergence the eyes move in opposite directions (at least for targets aligned at different depths along the median plane). The duration of vergence is generally longer than that of a saccade: typically lasting 250 to $300 \mathrm{~ms}$; its velocity is lower and ranges from $10^{\circ}$ to $30^{\circ}$ per second (e.g. [2]. By contrast, the latency of vergence is similar to that of a saccade, due to an almost identical neuronal circuit controlling their programming [2-4].

Combined eye movements are believed to be executed by the association of the saccade and of the vergence generators located at the brainstem [4]. Such movements are the most frequent movements we make in everyday life as the objects we explore in the real 3D space are located both in different directions and depths. For combined eye movements, the latency of the saccade or of the vergence component increases only slightly relatively to that of the saccade or vergence made alone. However, the velocity of vergence component is considerably increased relative to that of vergence alone $[3,4]$.

Today, two theories explaining the combined movements exist. The first suggests a combination of two motor commands at the brainstem subtending the components saccade and vergence components. The second suggests combined movements could be monocular saccade commands: such unequally sized saccades for each eye would increase or decrease the vergence angle of the optic axes appropriately for the depth at which the target is located [5]. Some evidence in line with the first, saccade-vergence interaction hypothesis occurring even at the cortical level comes from EEG studies in humans [6,7]. Cerebral EEG activity during the latency period was found to be widespread over the anterior central and posterior cortical areas. For convergence and divergence targets, this activity was bilaterally distributed; convergence targets activated a rather extended cortical network in the central and posterior area, while divergence targets activated a more confined posterior area, spreading ventrally from the occipital cortex. Interestingly, cortical activity for combined targets was lateralized contralateral to the stimulus but its topography resembled more closely to that before divergence stimulus. In primates [8], the existence of a high correlation between saccadic and vergence target selection was found suggesting that the amplitude computation process of both subsystems is due to a 
Citation: Kapoula Z, Pain E (2020) Differential Impact of Sound on Saccades Vergence and Combined Eye Movements: A Multiple Case Study. J Clin Stud Med Case Rep 7: 095.

common target selection stage that has access to information about stimulus location in 3-D space.

Let us now consider the multisensory aspects. It is known that vision and hearing work in cooperation in natural life. In the midbrain this is translated anatomically by overlapping visual and auditory receptive fields of neurons in the superior colliculus that mediate multisensory integration [9]. In the primary visual cortex, neurons responding to visual stimulation are connected with areas of the brain that are not specific to vision, allowing primary visual cortex response even in the absence of visual stimulation. Some of these connections are made with the auditory cortex [10]. EEG and fMRI studies have shown that auditory stimulation modulates the topography as well as the specificity of the activation of the primary cortex [11]. In addition, fMRI records showed that auditory stimulation alone activates the visual cortex (V1, V2, and V3) specifically for certain types of stimuli and can induce visual illusions when associated with certain visual stimulations $[12,13]$.

Returning to eye movements, the effect of auditory stimulation has been studied for saccades. Using visual or auditory stimulations at different lateral eccentricities Zambarbieri, et al. [14], and Zambarbieri, et al. showed shorter latency, higher speed and better accuracy for saccades to visual targets than for saccades to auditory targets [15]. Colonius and Arndt, et al. investigated the impact of a sound presented earlier or near simultaneously at a position near or far from that of the visual target position [16]. Their results on saccade latency indicate that the combination of visual and auditory targets involves intersensory facilitation. Such facilitation was mainly reflected in a reduction in the latency of saccades, particularly when the sound occurred $30 \mathrm{~ms}$ before the onset of the visual target; latency reduction was observed even when the distances between the auditory and visual stimulation position were as large as 45 to 55 ; the maximal decrease in latency was $63 \mathrm{~ms}$ occurring for the smaller distance, such as 5 between the visual and the auditory positions.

To date, no studies have examined the effect of auditory signal on vergence or combined saccade vergence movements. In view of the results reported for the impact of a sound on the latency of saccades to visual targets, we could expect similar, if not greater, effects for vergence. Yet, there is a basic difference between vergence and saccades, which is related to their duration and their mode of execution. Saccades are rather ballistic, open-loop movements, as they last, in their majority, less than $100 \mathrm{~ms}$. In contrast, vergence along the median plane can last several hundred milliseconds $[3,4]$; such movements are presumably controlled by both, a phasic programmed component and a visually driven component. During the latter, the visual system should evaluate the residual binocular disparity and keep the movement going on until the disparity becomes zero [17,18]. In this context, one would expect that vergence eye movements could be differently influenced by the auditory signal, i.e., possible effects on the duration of the movement itself. Thus, we hypothesized a differential effect of the sound corresponding to differential mode of control of the saccade and vergence systems. For combined eye movements that involve complex interaction of the saccade and vergence ocular motor subsystems one would expect effects of the sound that might be different from those for saccades and vergence made alone. The study also has a clinical motivation, namely, identifying factors that could accelerate the execution of vergence, as those movements are frequently inefficient in the clinical setting [2].

\section{Methods}

\section{Participants}

A total of 14 participants, 6 women and 8 men (mean age: 22.6 $+/-0.62$ years) were recruited for this protocol. Most of them were students from the neighbor laboratories. None of the participants showed oculomotor disorder or other health problem that could alter the results. An optometrist member of the team, conducted a verification of the visual health of each participant. No participant presented strabismus, amblyopia or any neuro-ophthalmologic disorder. They all had normal binocular vision (60 sec or arc or better, tested with the TNO stereovision test). No participant had vergence abnormalities (the proximal punctum of convergence was equal or less than 6 $\mathrm{cm}$ for all participants). No participant had back pain or equilibrium problems either; they all had some sport activity at the university. The investigation adhered to the tenets of the Declaration of Helsinki and was approved by the CNRS ethics committee (Comité de Protection des Personnes Sud Mediterranée III No. 186). Written informed consent was obtained from all the participants after the nature of the procedure was explained.

\section{Oculomotor tests: apparatus and stimuli}

The Remobi device (Réhabilitationde la MotricitéBinoculaire) was used to test saccades, vergence and combined eye movements (patent US8851669, WO2011073288). This device consists of a trapezoidal surface $150 \mathrm{~cm}$ long, upon which are embedded 48 LEDs, displayed at 4 arcs of isovergence located at $20 \mathrm{~cm}, 40 \mathrm{~cm}, 70 \mathrm{~cm}, 150 \mathrm{~cm}$ of the participant's eyes; at each arc LEDs were displayed at $0^{\circ}$, and at $5^{\circ}, 10^{\circ}, 15^{\circ}, 20^{\circ}$ to the left or to the right. Each LED emits at a wavelength of $626 \mathrm{~nm}$. Adjacent to the location of each LED there is a buzzer allowing an auditory stimulus specific to each location; the buzzer characteristics were as follows: nominal frequency approximately $2048 \mathrm{~Hz}$, sound pressure level $75 \mathrm{~dB}$, diameter $12 \mathrm{~mm}$.

The EyeSeeCam head-mounted video-oculography device was used to record the eye movements during the various tests. The headset consists of two infrared cameras and a laser pointer for calibration (see http://www.eyeseetec.de/eyeseecam-sci/).The sampling rate of the EyeSeeCam system was $220 \mathrm{~Hz}$. The optimal spatial resolution was approximately 0.01 degrees. Prior to testing, the EyeSeeCam system was calibrated using a laser beam to emit 5 points at the top, bottom, left and right of the participant's line of sight.

All tests were performed in a quiet experimental room with no specific sound isolation; the sound of each buzzer was distinctive and strong (about $75 \mathrm{~dB}$ ), and together with the visual LED target was a powerful stimulus. The participants were sitting in the dark with the EyeSeeCam device on the head, and the Remobi device was centered, at eye level; the first LED array was at $20 \mathrm{~cm}$ from the participant's eyes (Figure 1A). No head or chin rest was used. The ocular motor tests are described below.

Vergence test: For each trial the fixation LED $\left(0^{\circ}\right)$ light up at $40 \mathrm{~cm}$ for a period varying from $1400 \mathrm{~ms}$ to $2000 \mathrm{~ms}$. It was followed randomly by the target LED during $2000 \mathrm{~ms}$, appearing always at the center $\left(0^{\circ}\right)$ either at $20 \mathrm{~cm}$ (calling for a convergence movement of $8^{\circ}$ ) or $150 \mathrm{~cm}$ (calling for a divergence movement of $7^{\circ}$ ). The test contained 40 trials (20 trials of convergence, 20 of divergence, Figure 1A and 1C left). 
Citation: Kapoula Z, Pain E (2020) Differential Impact of Sound on Saccades Vergence and Combined Eye Movements: A Multiple Case Study. J Clin Stud Med Case Rep 7: 095.

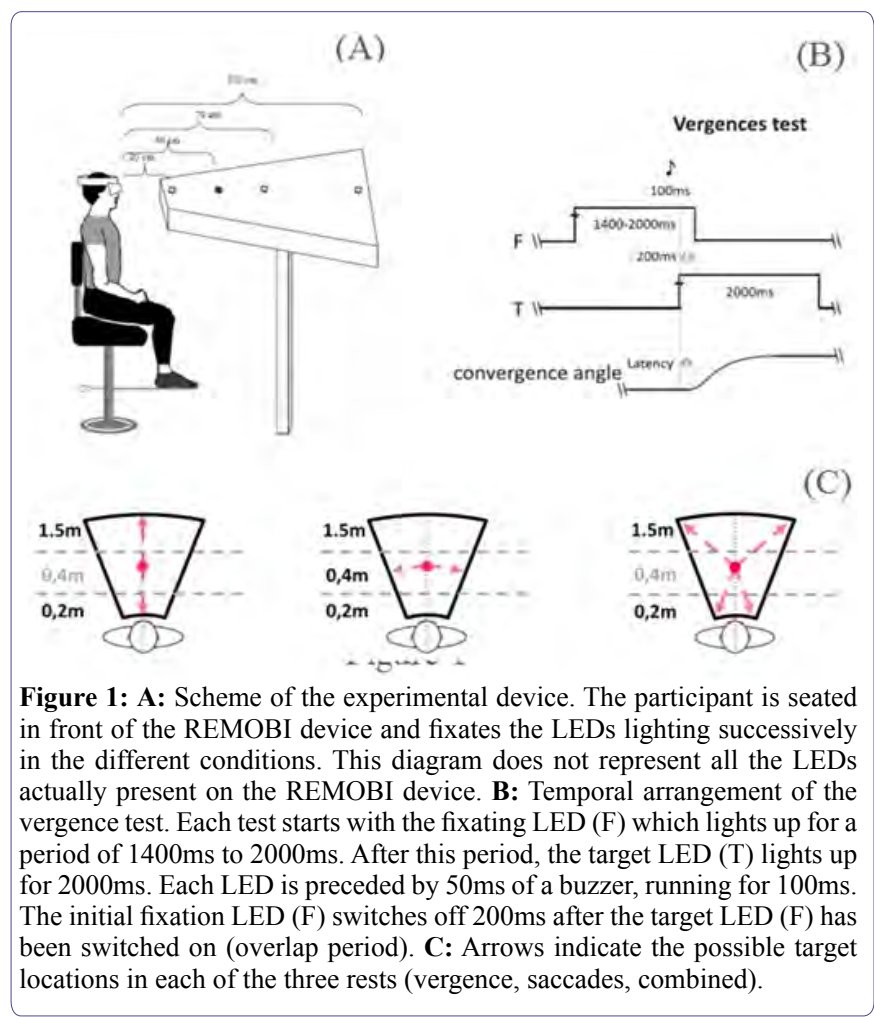

Saccade test: Each trial started with the fixation central LED lighting at $40 \mathrm{~cm}$ from the subject for a randomized period ranging from $1400 \mathrm{~ms}$ to $2000 \mathrm{~ms}$; it was followed by the lighting of the saccade target LED for $2000 \mathrm{~ms}$ at $20^{\circ}$ of eccentricity, randomly chosen on the left or on the right. There were 40 trials (20 left, 20 right, Figure 1C middle).

Combined saccade vergence test: At the beginning of each trial the fixation central LED light up at $40 \mathrm{~cm}$ from the participant's eyes for a period varying from 1400 to $2000 \mathrm{~ms}$. It was followed by the lighting of the target LED for $2000 \mathrm{~ms}$ randomly at a depth distance of $20 \mathrm{~cm}$ or $150 \mathrm{~cm}$ and at $20^{\circ}$ left or right. The test contained 80 trials (20 convergent left trials with the target appearing at $20^{\circ}$ to the left at $20 \mathrm{~cm}$ from the subject's eyes, 20 divergent trials to the left with the target appearing at $20^{\circ}$ left at $150 \mathrm{~cm}$ distance, 20 convergent trials to the right, with the target being at $20^{\circ}$ right and at $20 \mathrm{~cm}$ depth, and 20 divergent trials to the right with the target at $20^{\circ}$ right and at $150 \mathrm{~cm}$ depth (Figure 1C, right).

For all three tests, the central fixation LED was switched off 200 ms after the lighting of the target LED (overlap paradigm, Figure 1B). The blue lines in Figure 3 indicate the possible target locations for each of the tests. Each of the three tests (saccades, vergence, combined) was performed twice, with or without auditory signal preceding the target LED (auditory-visual test, visual test). For auditory-visual tests the buzzer adjacent to the initial fixation LED emitted a sound for $100 \mathrm{~ms}$ duration starting $50 \mathrm{~ms}$ before the onset of the LED (Figure 1B). Fifty milliseconds before the onset of the next target LED, the buzzer next to the target LED emitted a sound for $100 \mathrm{~ms}$ duration and similarly for other target LEDs.

For all eye movement tests, the participants were instructed to respond to the target LED as soon and as accurately as possible.
For visual tests, the target LED light alone and the buzzer was kept silent. To counter the fatigability, the order of the tests (visual, auditory-visual) was alternated for different participants.

(A)

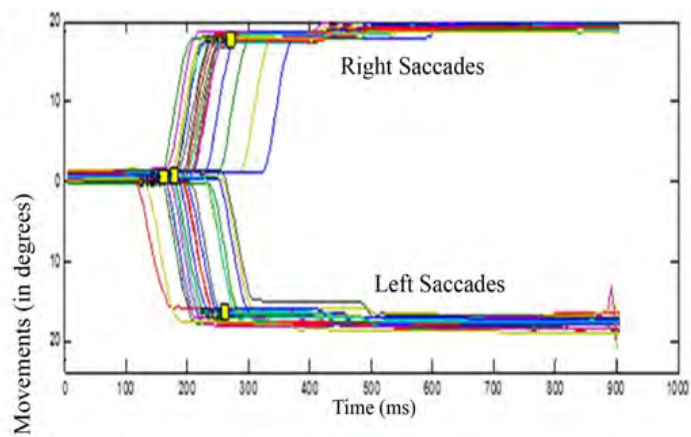

(B)

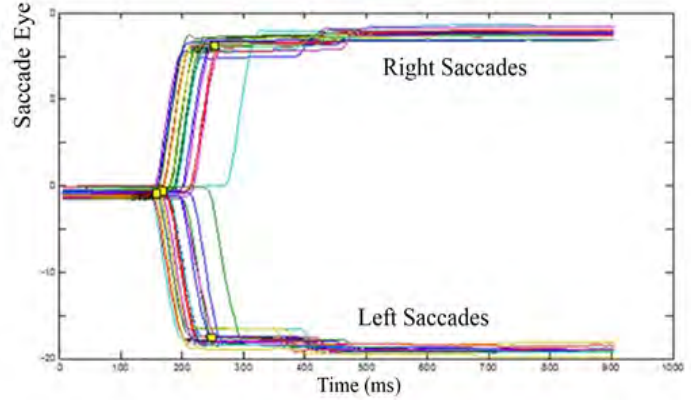

Figure 2: Trajectories of the saccades of a participant (in degrees) as a function of time (in ms): ocular saccades tothe right or to the left for visual targets (A) and for auditory-visual targets (B).

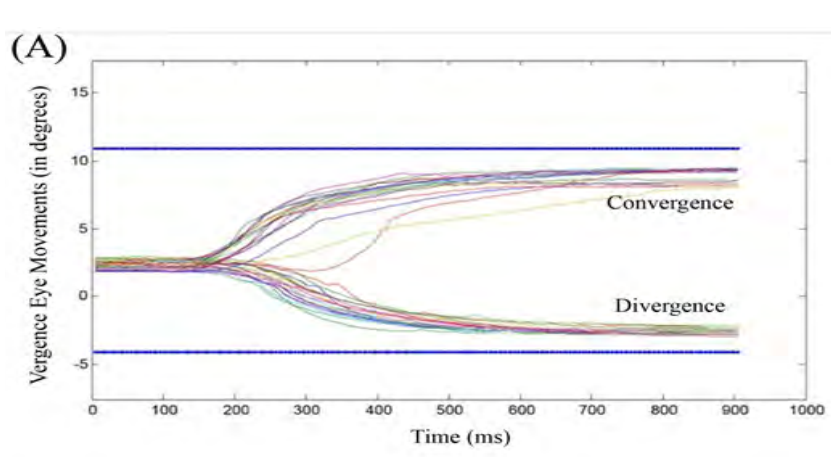

(B)

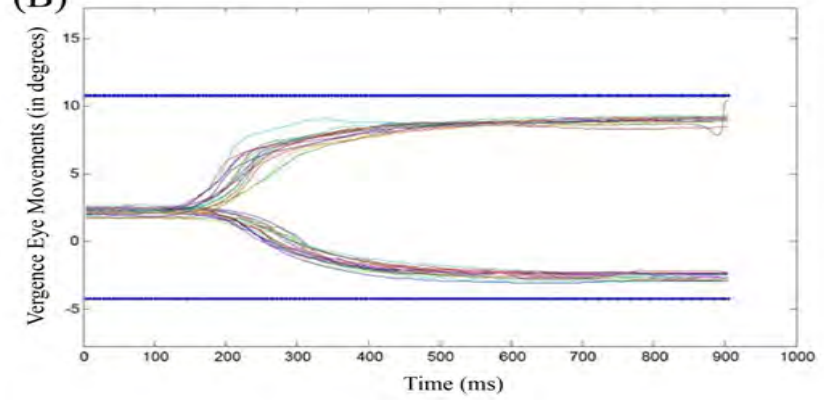

Figure 3: Trajectories of convergence (upward inflexion) and divergence (downward inflexion) for visual targets (A) and for auditory-visual targets (B). The blue lines indicate the target amplitude. 
Citation: Kapoula Z, Pain E (2020) Differential Impact of Sound on Saccades Vergence and Combined Eye Movements: A Multiple Case Study. J Clin Stud Med Case Rep 7: 095.

Sound characteristics and purpose: The 2-kHz tone of the buzzer loudspeaker (sound pressure $75 \mathrm{~dB}$, diameter $12 \mathrm{~mm}$ ), served as a carrier for the transient warning sound. This sound was a short, 100-ms burst with a sharp onset (triggered by a rectangular window). The performance in terms of lateral localization (for the saccade task) is mainly determined by the ability of the listener to exploit the interaural time difference [19], between the two ears, determined by the sharp onset of the warning sound. In terms of distance in depth (for the vergence task), the performance is determined by the level of the warning sound. In both cases, the carrier frequency bears little influence on the performance because what matters is the onset delay or the loudness while spectral cues are not used in our experiment.

\section{Data analysis and statistics}

The data generated by the EyeSeeCam were analyzed with the software developed in the laboratory (AIDEAL, patent 2004768 submitted, DSO2020003510). The software calculated the latency, the duration, the amplitude, and the mean velocity for each movement using the following criteria: the beginning of the saccade was determined when the eye velocity exceeded $10 \%$ of its maximum velocity and the end of the movement was determined when the eye velocity returned below $10 \%$. Similar criteria have been used in our prior studies and by others $[20,21]$. Vergence was measured as the difference of the right eye instantaneous position from that of the left eye position. The beginning and the end of the vergence was determined when the velocity of the vergence signal exceeded and returned to $5 \% \mathrm{~s}$, respectively. Such criteria have been also used in prior studies [2-3,22-24]. The mean velocity was measured as the ratio of the amplitude of the movement (in degrees) over its duration (in seconds). To evaluate the accuracy of the movement we calculated the gain, i.e. the ratio of the amplitude of the movement over the amplitude of the target requirement. Saccades to the left and to the right were analyzed separately as at the individual level some left-right asymmetries are known to exist albeit idiosyncratic [25]. Combined eye movements were broken into their saccade and vergence components respectively and were analyzed using similar criteria and parameters as those for the saccade or vergence tests alone (latency, duration, mean velocity, gain). There were thus four components: convergence with a saccade component to the left or to the right; divergence with a saccade component either to the left or to the right.

Some eye movements were rejected from the analysis due to blinks or to partial loss of signal during the recording, or to anticipation (i.e., below $80 \mathrm{~ms}$ ) or to latencies longer than $800 \mathrm{~ms}$. These criteria of exclusion are standard $[23,24,26]$.

Because of the relatively limited number of participants and the non-normality of distribution of certain parameters of eye movements, statistical analyses of each parameter were performed by Wilcoxon's nonparametric tests (STATISTICA 7). Analysis of the 14 participants was possible for all parameters of the vergence movements along the median plane; however, for saccades or combined eye movements involving $20^{\circ}$ of eccentricity the recordings could be noisy for some participants or the eye tracker signal was lost. For combined eye movements statistical analysis was also done separately for left and right saccade components combined with either convergence or divergence. All parameters were first averaged per individual, and then tested for group differences between conditions.

\section{Results}

\section{Saccades to visual vs auditory-visual targets}

Figures $2 \mathrm{~A}$ and $2 \mathrm{~B}$ show the trajectories of saccade movements; A depicts movements with only visual targets, B movements with auditory-visual targets; upward inflexion represents rightward saccades, and downward leftward saccades. Qualitatively, the two conditions show relatively similar ocular saccades. However, there is one clear difference: The addition of the sound substantially reduces the latency of the saccades towards shorter values.

Quantitative data (group means and standard deviations) are shown in Table 1; statistical analysis completed on 12 of the 14 participants indicated a significant difference for the mean latency between the two conditions: for visual targets alone, the average latency for leftward saccades was $200 \mathrm{~ms}$, while for auditory-visual targets it dropped to $190 \mathrm{~ms}(\mathrm{Z}=2.5 ; \mathrm{p}=0.01)$. For rightward saccades to visual targets the latency was $203 \mathrm{~ms}$; for auditory-visual targets it dropped to $191 \mathrm{~ms}(Z=2.05 ; \mathrm{p}=0.04)$. No other statistical differences were found; the values of average velocity shown in Table 1 were not statistically significant. We also measured peak velocity (not shown in Table 1) and this was also not statistically significant: saccades to the left had an average peak velocity of $429 \%$ s for visual targets vs $435 \%$ for auditory visual targets $(\mathrm{Z}=1.53, \mathrm{p}=0.12)$; for rightward saccades the mean peak velocity was $432 \%$ s for visual target, and 436 $\%$ s for auditory visual targets $(Z=1.66, p=0.09)$.

\section{Vergence to visual vs auditory-visual targets}

Figures $3 \mathrm{~A}$ and $3 \mathrm{~B}$ show vergence movements to visual and auditory-visual targets (A, B, respectively). The Figure shows differences indicating better performance for the condition with auditory-visual targets. Particularly, the movements of convergence (upward inflexion) and divergence (downward inflexion) are both faster in execution (their slopes are sharper).

Group means and their standard deviations are shown in Table 1. The Wilcoxon test was performed on all participants, comparing each parameter under conditions visual vs auditory-visual targets. The results showed statistically significant differences for two parameters: the duration and the mean velocity. For visual targets alone, the average duration of the divergence was $309 \mathrm{~ms}$, and it dropped to $284 \mathrm{~ms}$ for auditory-visual targets. For convergence, the duration was $274 \mathrm{~ms}$ for the visual targets and dropped to $236 \mathrm{~ms}$ for auditory-visual targets. The difference in duration was statistically significant $(25 \mathrm{~ms}$ for divergence, $Z=2.63 ; \mathrm{p}=0.009 ; 38 \mathrm{~ms}, \mathrm{Z}=2.67 ; \mathrm{p}=0.0076$ for convergence). The mean velocity of divergence increased from $15 \% \mathrm{~s}$ for visual targets to $16 \%$ s for auditory-visual targets, and the mean velocity of convergence from $23 \%$ s to $27 \%$ s for auditory-visual targets. The difference for the mean velocity was also statistically significant at $1 \%$ s for divergence $(Z=2.58 ; p=0.009)$ and $4 \% \mathrm{~s}(Z=2.40$; $\mathrm{p}=0.016$ ) for convergence. In contrast, the latency of divergence was higher for auditory-visual targets relative to visual targets (from $206 \mathrm{~ms}$ to $225 \mathrm{~ms}$ ); this difference of $19 \mathrm{~ms}$ was statistically significant $(\mathrm{Z}=2.17 ; \mathrm{p}=0.03)$. Interestingly the statistical analysis on the peak velocity (not shown in Table 1) did not show any significant differences: the mean peak velocity of convergence to visual targets was $62 \%$ s vs $69^{\circ} / \mathrm{s}$ for auditory-visual targets $(\mathrm{Z}=1.44, \mathrm{p}=0.148)$; for divergence the mean peak velocity to visual targets was $38^{\circ} / \mathrm{s}$ vs $39^{\circ} / \mathrm{s}$ for auditory-visual targets $(Z=1.64, p=0.09)$. 
Citation: Kapoula Z, Pain E (2020) Differential Impact of Sound on Saccades Vergence and Combined Eye Movements: A Multiple Case Study. J Clin Stud Med Case Rep 7: 095.

\begin{tabular}{|c|c|c|c|c|c|c|c|c|c|}
\hline & & \multicolumn{2}{|c|}{ Latency (ms) } & \multicolumn{2}{|c|}{ Duration (ms) } & \multicolumn{2}{|c|}{ Mean velocity (Degrees/s) } & \multicolumn{2}{|c|}{ Gain } \\
\hline & & Visual & $\begin{array}{c}\text { Audito- } \\
\text { ry-visual }\end{array}$ & Visual & $\begin{array}{l}\text { Audito- } \\
\text { ry-visual }\end{array}$ & Visual & $\begin{array}{l}\text { Auditory-vi- } \\
\text { sual }\end{array}$ & Visual & $\begin{array}{l}\text { Audito- } \\
\text { ry-visual }\end{array}$ \\
\hline & & & & $\begin{array}{c}\text { Mean }+/ \text { - Standard } \\
\text { Deviation }\end{array}$ & & & & & \\
\hline \multirow{2}{*}{ Vergence } & Convergence (14) & $176+/-29$ & $189+/-32$ & $274+/-57$ & $236+/-50 *$ & $23+/-4$ & $27+/-5 *$ & $0.8+/-0.7$ & $0.7+/-0.6$ \\
\hline & Divergence (14) & $206+/-33$ & $225+/-41 *$ & $309+/-52$ & $284+/-45^{*}$ & $15+/-2$ & $16+/-2 *$ & $0.7+/-0.4$ & $0.7+/-0.4$ \\
\hline \multirow{2}{*}{ Saccade } & Right (12) & $203+/-32$ & $191+/-35 *$ & $71+/-4$ & $70+/-4$ & $247+/-15$ & $206+/-21$ & $0.88+/-0.1$ & $0.89+/-0.1$ \\
\hline & Left (12) & $200+/-37$ & $190+/-34 *$ & $72+/-4$ & $71+/-6$ & $246+/-20$ & $251+/-18$ & $0.73+/-0.1$ & $0.89+/-0.1$ \\
\hline Combined Convergent & Saccade (10) & $251+/-40$ & $236+/-36$ & $68+/-6$ & $64+/-5^{*}$ & $229+/-22$ & $229+/-21$ & $0.76+/-0.1$ & $0.72+/-0.1^{*}$ \\
\hline Movement to the Right & Vergence (10) & $242+/-39$ & $220+/-32 *$ & $77+/-14$ & $95+/-24$ & $64+/-13$ & $66+/-13$ & $0.7+/-0.6$ & $0.7+/-0.6$ \\
\hline Combined Convergent & Saccade (10) & $252+/-41$ & $230+/-39 *$ & $67+/-5$ & $70+/-5$ & $221+/-21$ & $249+/-21 *$ & $0.81+/-1$ & $0.86+/-0.1$ \\
\hline Movement to the Left & Vergence (10) & $275+/-42$ & $252+/-40 *$ & $78+/-15$ & $74+/-15$ & $75+/-14$ & $77+/-18$ & $0.7+/-0.7$ & $0.7+/-0.5$ \\
\hline Combined Divergent & Saccade (10) & $250+/-45$ & $248+/-43$ & $76+/-6$ & $77+/-6$ & $249+/-19$ & $261+/-21$ & $0.90+/-0.1$ & $0.92+/-0.2$ \\
\hline Movement to the Right & Vergence (10) & $254+/-46$ & $258+/-41$ & $188+/-28$ & $156+/-29$ & $29+/-6$ & $28+/-6$ & $0.6+/-0.5$ & $0.6+/-0.5$ \\
\hline Combined Divergent & Saccade (10) & $253+/-45$ & $230+/-38 *$ & $81+/-5$ & $76+/-5 *$ & $242+/-17$ & $240+/-19$ & $0.94+/-0.1$ & $0.98+/-0.1 *$ \\
\hline Movement to the Left & Vergence (10) & $262+/-45$ & $239+/-44$ & $138+/-24$ & $165+/-30$ & $31+/-8$ & $30+/-6$ & $0.7+/-0.5$ & $0.7+/-0.5$ \\
\hline
\end{tabular}

Table 1: For each type of movement is shown the group mean and the standard deviation. The mean velocity is the ratio of amplitude to duration. The accuracy is characterized by the gain value i.e. the ratio of the amplitude of the movement to the amplitude of the target. A gain value of 1 shows optimal accuracy. Asterisks indicate statistically significant differences between the movements to visual and to auditory-visual targets.

This means that the sound had an effect mostly on the second deceleration phase of the velocity profile of vergence and its kinematics measured by the average velocity. In summary, the changes due to the addition of the auditory signal were made on the duration and average velocity for the vergence but on the latency for the saccades.

\section{Combined movements to visual vs auditory-visual targets}

As mentioned, combined eye movements were split into their vergence and saccade component; the statistical analysis (comparing auditory-visual vs visual tests) was made separately for the four components: convergence with a saccade component to the left, convergence with a saccade component to the right, divergence with a saccade component to the left or to the right. Figure 4 shows examples of trajectories of convergent combined movements to the left: A, B show the saccade and convergence components for visual targets, $\mathrm{C}$ and $\mathrm{D}$ show saccade and convergence components for auditoryvisual targets. The saccade component to the auditory-visual targets had lower latency and faster execution (the slope of the trajectory is sharper, Figure 4A vs 4C).

Quantitative group mean results for each component of the combined eye movements are shown in Table 1. Statistical analyses were carried on 9 of the 14 participants. For divergent movements to the left, the latency of the saccade component was significantly lower for the condition with auditory-visual targets $(253 \mathrm{~ms}$ to $230 \mathrm{~ms} ; \mathrm{Z}=2.66$; $\mathrm{p}=0.007)$; the duration was also lower for auditory-visual than for visual targets, $81 \mathrm{~ms}$ vs $76 \mathrm{~ms} \mathrm{(} \mathrm{z}=2.07 ; \mathrm{p}=0.03)$. Finally, the gain (the ratio of movement amplitude over target amplitude) was also higher for the auditory-visual than for the visual targets ( 0.94 to $0.98, Z=2.43$; $\mathrm{p}=0.01$ ). The convergent movements to the left also showed significant differences between the auditory-visual and the visual targets: the latency of the saccade component was lower by $22 \mathrm{~ms}(\mathrm{Z}=2.10 ; \mathrm{p}$ $=0.03)$, and the mean velocity was higher by $28 \% \mathrm{~s}(249 \% \mathrm{~s}$ vs $221 \%$ s, $\mathrm{Z}=1.96, \mathrm{p}=0.04)$.

In contrast, for combined movements to the right statistically significant differences were observed only for the convergent movements, namely the duration of the saccade component was lower by $4 \mathrm{~ms}$ for auditory-visual targets $(\mathrm{Z}=2.52 ; \mathrm{p}=0.01)$ but the gain for auditory-visual targets was significantly lower than for visual targets alone ( 0.76 vs $0.7, Z=2.31, p=0.02$ ). The latency of the convergence component was significantly shorter for auditory-visual targets ( $220 \mathrm{~ms}$ vs $242 \mathrm{~ms}, \mathrm{p}=0.01$ ). For divergent movements to the right no significant differences were observed for either component.

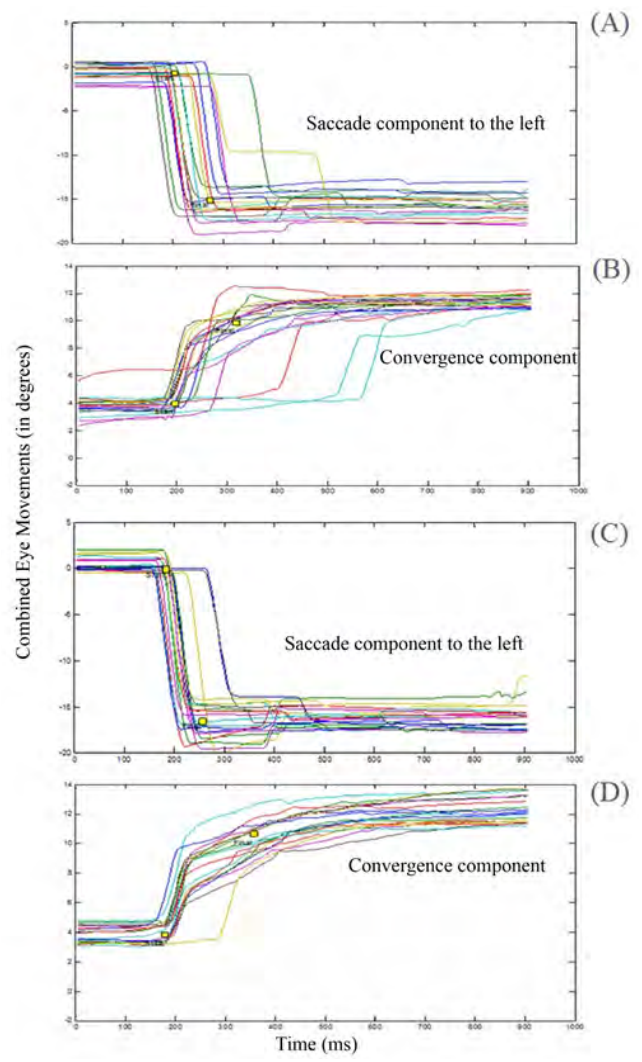

Figure 4: Convergent combined movements to the left for visual targets (A, B) and for auditory-visual targets $(\mathbf{C}, \mathbf{D})$; their saccade component is shown in $\mathrm{A}$ and $\mathrm{C}$, respectively and their convergence component in $\mathrm{B}$ and $\mathrm{C}$. 


\section{Synthesis of main sound effects}

The addition of the sound decreased the latency of saccades regardless of their direction; for vergence it decreased the duration and increased the mean velocity for both convergence and divergence. For convergent or divergent combined movement the sound effects were more prominent for the leftward direction, specifically for their saccade component (latency and duration decreased, mean velocity increased). For combined movements to the right the addition of the sound caused considerable differences only for convergent movements: the duration of the saccade component was shorter for auditory-visual targets than visual targets, and the latency of the convergence component was shorter.

\section{Discussion}

\section{Saccades}

In the present study we investigated left and right saccades separately as there is prior literature suggesting some idiosyncratic differences between left and right movements [25]. The results indicate that the parameter most modified by the addition of the sound stimulation is the latency of the saccades. These results are in line with the literature, which showed a decrease in latencies for auditory-visual targets [16]. The mechanism of such decrease of latency could be intersensory attentional alerting and multisensory integration improving target localization and saccade programming. We found no effect on the other parameters of the saccades, as neither the duration, the mean velocity, the peak velocity nor the gain were modified by the addition of the sound.

We now consider the targets in depth requiring vergence eye movements. Prior research on targets in depth exist but with no recording and analysis of vergence eye movements. These studies will be briefly summarized. Van der Stoep, et al. investigated reaction times to lateral auditory-visual targets; in some cases the auditory signal was presented at the same eccentricity and the same depth as the visual signal while in other cases the sound was presented at the same eccentricity but at a different depth. Cross-modal auditory visual facilitation was only observed in the former case, indicating that cross-modal attention is depth aware [27]. In another study it was shown that the correction of the targets for the depth (decrease of the sound intensity for targets at far, or decrease of their angular size) diminishes cross modal facilitation relative to when stimuli are not corrected for depth [28]. In our study, auditory-visual stimuli were presented at the same depths every time, and there were 3 possible depths (initial fixation at a depth of $40 \mathrm{~cm}$, target location at $20 \mathrm{~cm}$ or $150 \mathrm{~cm}$ in depth) and vergence eye movements were recorded and analyzed for the first time. In a prior study, Van der Stoep, et al. investigated the Stimulus Onset Asynchrony for auditory-visual with values varying from $0,50,100$ and $200 \mathrm{~ms}$ [29]; their results indicated that the $50 \mathrm{~ms}$ SOA produced the most enhanced multisensory responses, attributed to cross-modal exogenous spatial attention and multisensory integration. In the present study, we also used a 50ms SOA. Next are discussed the results on vergence eye movements along the median plane or combined with saccades.

\section{Vergence}

The results show for the first time that a sound delivered just before a target for vergence increases the velocity and decreases the duration of the movement but not its latency. The localization of the sound is mainly derived from the interaural difference in the intensity of the sounds and from the time difference of arrival of the sounds [30]. For vergence along the median plane, there is no interaural difference of intensity. Yet, the addition of monaural auditory cues could have reduced the latency of vergence, as was the case for the saccades. The observed substantial changes in only the duration and the mean velocity suggest a different mechanism of action of the sound for vergence movements. The peak velocity which is achieved early during the trajectory of the vergence remained the same for the two types of the targets, but the average velocity increased for auditory-visual targets. This indicates that the kinematics of the vergence changed, namely the shortening of vergence duration was due to shortening of the deceleration phase. Similar observations have been made in the past for modifications of vergence profiles with age [31]. Also, our observations are in line with the study [32], indicating that sound can have effects on the kinematics of eye movements, in our case shortening the deceleration phase of vergence. Plausibly the auditory signal acts more downstream, at the level of execution of the movement; for instance, at the level of multisensory neurons in the superior colliculus [8], which would in turn stimulate the vergence generator cells in the mesencephalic reticular formation responsible for the generation of the motor command. However, it is also possible that the auditory and oculomotor signals could interact at the cortical level including at the visual parietal and frontal cortices known to process continuously the residual disparity to control the ongoing execution of the vergence, i.e. the closed loop part of the vergence see model [18]; their important communication during audio-visual stimulation could directly induce a higher stimulation of the superior colliculus to relay a stronger signal to the brainstem vergence generator [9-11].

Statistical analysis also revealed an increase in the latency for auditory-visual targets, but only in divergence. The divergent movement is different from the convergent movement as its onset includes a mechanism of release of the previous convergence that presumably could intervene early in the divergence initiation process. The presence of the auditory signal could delay such release process, resulting in the increase of the latency observed. These differences between vergence and saccades for auditory-visual stimuli, confirm that convergence, divergence and saccades are underpinned by distinct cortical-subcortical sub-circuits, as suggested by Yang et al. [3]. They are programmed differently, each involving many specific relays and reacting uniquely to the multisensory integration of an auditory signal together with the visual signal.

\section{Combined eye movements}

Combined eye movements are known to be characterized by an acceleration of the vergence by the saccade $[3,4,33]$. In line with such prior studies, our data on the duration and velocity of the vergence components compared with those of vergence alone clearly show much faster vergence dynamics in the former case (Table 1); as an example, the duration of the convergence alone versus combined, in the no sound condition decreased from $274 \mathrm{~ms}$ to $78 \mathrm{~ms}$, and the duration of divergence decreased from $309 \mathrm{~ms}$ to $163 \mathrm{~ms}$. Next, we will discuss the effects of sound to such movements.

It is important that most of the statistically significant differences due to sound occurred for leftward movements. The latency of the leftward saccade component combined with either convergence or 
Citation: Kapoula Z, Pain E (2020) Differential Impact of Sound on Saccades Vergence and Combined Eye Movements: A Multiple Case Study. J Clin Stud Med Case Rep 7: 095.

divergence, was substantially lower for auditory visual targets than for visual targets. For rightward combined movements substantial latency decrease occurred only for the convergence component. Another important aspect is that for the leftward movements combining saccade and vergence, the effect of the sound was found to be different than that for saccade or vergence movements made alone. Indeed, in addition to the latency decrease, the sound caused reduction in the duration of the saccade component or an increase of its mean velocity. In other words, the saccade components of the leftward combined movements are influenced by the sound in both their latency (decrease) and their dynamics (increase of average velocity and decrease of its duration). This preponderance of sound effects for leftward combined movements could be related to attentional bias: left-right asymmetries have been previously observed, for instance in perceptual judgment tasks that are most likely due to the right hemisphere dominance for spatial attention $[34,35]$. Noteworthy, for saccades made alone latency decrease was observed for both left and right saccades. Thus, taken together, the combination of a saccade to left with vergence could be facilitated by multisensory integration and intersensory attentional alerting.

That the sound effects are different for the saccade and vergence components of the combined eye movements than for the saccade and vergence made alone, and their leftward preponderance, are new observations supporting the hypothesis of complex interaction between the saccade and vergence systems both at the cortical and subcortical level $[3,4,7,16]$. The alternative hypothesis mentioned in the Introduction, according to which combined eye movements would be monocular saccade commands would predict similar effects of the sound for saccades and combined eye movements and this not the case in our observations [11].

\section{More than a warning effect - sound acts of continuous visu- al processing of targets in depth}

In this study, we used the overlap paradigm for all testing conditions (visual or auditory visual targets): the target LED appeared before extinguishing the central fixation LED (with an overlapping period of 200ms). Another well-known paradigm is the gap, in which the fixation LED is extinguished prior (typically $200 \mathrm{~ms}$ ) to the onset of the target LED. Such early extinction has been found to act as a warning signal as it systematically reduces the latency of both saccades and vergence [20,31,36,37]. One could argue that the sound prior to the target LED simply acted as a warning signal in a similar way as the switching off of the fixation LED in the gap paradigm. If such was the case the sound should reduce the latency of both saccades and vergence. But we observed latency decrease only for the saccades, not effect on convergence latency and a latency increase for divergence. Instead sound affected the duration and velocity of vergence, namely the deceleration phase of the vergence execution. The deceleration phase of vergence is under the control of visually driven feedback in a closed loop [18]. Our observations for vergence suggest a lasting sound effect on the visual processing of the target in depth that shortens the duration of the deceleration phase of the vergence. This interpretation is in line with what we had proposed earlier for physiologic ageing of vergence eye movements, slowing down their visually driven component due to cerebral ageing [26]. More generally, the findings are in line with the studies reviewed in the Introduction, showing that auditory stimulation alone activates the visual cortex (V1, V2, and V3) [12].

\section{Conclusion}

In conclusion, the addition of a sound prior to a visual target, influences all types of eye movements but differently: it reduces the latency of saccades alone, the duration of convergence or divergence alone, and it reduces both the latency and the duration of the saccade component of leftward combined eye movements only. We hope that this study will stimulate further investigations, namely with the use of more target locations for saccades or vergence, or the use of different scales and types of sound. The study also has clinical implication, as it shows that multi sensorial stimulation, in this case auditory-visual, could speed up vergence along the median plane. As vergence disorders are frequent concerning more than 30 percent of the population, it is important for rehabilitation purposes to use auditory-visual stimulation, as was recently done by Kapoula et al. [2].

\section{Additional Information}

Author Contributions: Dr. Zoi Kapoula was responsible for the idea and the experimentation protocol, for writing the paper. Erwann Pain conducted the experiments and analyzed the data. We thank Akshay Chaturvedi for participating in some of the data analysis.

\section{Competing Interests}

The author(s) declare no competing interests.

\section{Acknowledgement}

The authors thank Pr. Paul Avan for discussions on the auditory stimuli. The research was supported by Fondation Avenir. The authors thank the anonymous reviewers for their insightful comments.

\section{Data Availability Statement}

The datasets generated during and analyzed during the current study are available from the corresponding author on reasonable request.

\section{References}

1. Bahill AT, Clark MR, Stark L (1975) The main sequence, a tool for studying human eye movements. Mathematical Biosciences 24: 191-204.

2. Kapoula Z, Morize A, Daniel F, Jonqua F, Orssaud C, et al. (2016) Objective Evaluation of Vergence Disorders and a Research-Based Novel Method for Vergence Rehabilitation. Transl Vis Sci Technol 5: 8.

3. Yang Q, Bucci MP, Kapoula Z (2002) The latency of saccades, vergence, and combined eye movements in children and in adults. Invest Ophthalmol Vis Sci 43: 29392949 .

4. Zee DS, Fitzgibbon EJ, Optican LM (1992) Saccade-vergence interactions in humans. J Neurophysiol 68: 1624-1641.

5. King WM (2011) Binocular coordination of eye movements--Hering's Law of equal innervation or uniocular control? Eur J Neurosci 33: 2139-2146.

6. Kapoula Z, Evdokimidis I, Smyrnis N, Bucci MP, Constantinidis TS (2002) EEG cortical potentials preceding vergence and combined saccade-vergence eye movements. Neuroreport 13: 1893-1897.

7. Tzelepi A, Lutz A, Kapoula Z (2004) EEG activity related to preparation and suppression of eye movements in three-dimensional space. Exp Brain Res 155: 439-449.

8. Chaturvedi V, Gisbergen JA (1998) Shared target selection for combined version-vergence eye movements,J Neurophysiol 80: 849-862. 
Citation: Kapoula Z, Pain E (2020) Differential Impact of Sound on Saccades Vergence and Combined Eye Movements: A Multiple Case Study. J Clin Stud Med Case Rep 7: 095.

9. Kadunce DC, Vaughan JW, Wallace MT, Stein BE (2001) The influence of visual and auditory receptive field organization on multisensory integration in the superior colliculus. Exp Brain Res 139: 303-310.

10. Petro LS, Paton AT, Muckli L (2017) Contextual modulation of primary visual cortex by auditory signals. Philos Trans R Soc Lond B Biol Sci 372: 20160104

11. Cappe C, Thut G, Romei V, Murray MM (2010) Auditory-visual multisensory interactions in humans: timing, topography, directionality, and sources. J Neurosci 30 : 12572-12580.

12. Vetter P, Smith FW, Muckli L (2014) Decoding sound and imagery content in early visual cortex. Curr Biol 24: 1256-1262.

13. Watkins S, Shams L, Tanaka S, Haynes JD, Rees G (2006) Sound alters activity in human V1 in association with illusory visual perception. Neuroimage 31: 1247-1256.

14. Zambarbieri D (2002) The latency of saccades toward auditory targets in humans. Prog Brain Res 140: 51-59.

15. Zambarbieri D, Schmid R, Magenes G, Prablanc C (1982) Saccadic responses evoked by presentation of visual and auditory targets. Exp Brain Res 47: 417-427.

16. Colonius H, Arndt P (2001) A two-stage model for visual-auditory interaction in saccadic latencies. Percept Psychophys 63: 126-147.

17. Erkelens CJ (2011) A dual visual-local feedback model of the vergence eye movement system. J Vis.11: 21.

18. Hung GK, Semmlow JL, Ciuffreda KJ (1986) A dual-mode dynamic model of the vergence eye movement system. IEEE Trans Biomed En 33: 1021-1028.

19. Yost WA (2017) Sound source localization identification accuracy: Envelope dependencies. J Acoust Soc Am 142: 173-185.

20. Vernet M, Yang Q, Gruselle M, Trams M, Kapoula Z (2009) Switching between gap and overlap pro-saccades: cost or benefit? Exp Brain Res 197: 49-58.

21. Eggert T, Robinson FR, Straube A (2016) Modeling Inter-trial Variability of Saccade trajectories: Effects of Lesions of the Oculomotor Part of the Fastigial Nucleus. PLoS Comput Biol 12: 1-33.

22. Coubard OA, Kapoula Z (2008) Saccades during symmetrical vergence. Graefes Arch Clin Exp Ophthalmol 246: 521-536.

23. Lang A, Gaertner C, Ghassemi E, Yang Q, Orssaud C, et al. (2014). Saccade-vergence properties remain more stable over short-time repetition under overlap than under gap task: A preliminary study. Front Hum Neurosci 8: 372.

24. Yang Q, Kapoula Z (2004) Saccade-vergence dynamics and interaction in children and in adults. Exp Brain Res 156: 212-223.
25. Honda H (2002) Idiosyncratic left-right asymmetries of saccadic latencies: Examination in a gap paradigm. Vision Res 42: 1437-1445.

26. Yang Q, Vernet M, Orssaud C, Bonfils P, Londero A, et al. (2010) Central crosstalk for somatic tinnitus: abnormal vergence eye movements. PLoS One 5: 11845.

27. Van der Stoep N, Nijboer TC, Van der Stigchel S (2014) Exogenous orienting of crossmodal attention in 3-D space: support for a depth-aware crossmodal attentional system,Psychon Bull Re 21: 708-714.

28. Van der Stoep N, Van der Stigchel S, Nijboer TC, Van der Smagt MJ (2016) Audiovisual integration in near and far space: effects of changes in distance and stimulus effectiveness. Exp Brain Res 234: 1175-1188.

29. Van der Stoep N, Spence C, Nijboer TC, Van der Stigchel S (2015) On the relative contributions of multisensory integration and crossmodal exogenous spatial attention to multisensory response enhancement. Acta Psychol (Amst) 162: 20-28.

30. Litovsky RY, Ashmead DH (1997) Development of binaural and spatial hearing in infants and children. In: Gilkey RH, Anderson TR (Eds.). Binaural and Spatial Hearing in Real and Virtual Environments. Mahwah, N.J.: Lawrence Erbaum Associates, Page no:571-592.

31. Yang Q, Le TT, Kapoula Z (2009) Aging effects on the visually driven part of vergence movements,Invest Ophthalmol Vis Sci 50: 1145-1151.

32. Frens MA, Van Opstal AJ (1995) A quantitative study of auditory-evoked saccadic eye movements in two dimensions. Exp Brain Res 107: 103-117.

33. Leigh RJ, Zee DS (2006) The Neurology of Eye Movements (4th edn). Oxford University Press, New York, Page no: 343-382.

34. Corbetta M, Shulman GL, Miezin FM, Petersen SE (1995) Superior parietal cortex activation during spatial attention shifts and visual feature conjunction Science 270: 802-805.

35. Niemeier M, Stojanoski B, Singh VW, Chu E (2008). Paradoxical cross-over due to attention to high or low spatial frequencies. Brain Cogn 67: 115-125.

36. Takagi M, Frohman EM, Zee DS (1995) Gap-overlap effects on latencies of saccades,vergence and combined vergence-saccades in humans,Vision Res 35: 3373 3388 .

37. Gaertner C, Wiener-Vacher S, Kapoula Z (2016) Up/down anisotropies of vertical saccades in healthy children depending on the mode and the depth of execution. Int J Dev Neurosci 52: 82-92. 


\section{di \\ Hramo}

Advances In Industrial Biotechnology | ISSN: 2639-5665

Advances In Microbiology Research | ISSN: 2689-694X

Archives Of Surgery And Surgical Education | ISSN: 2689-3126

Archives Of Urology

Archives Of Zoological Studies | ISSN: 2640-7779

Current Trends Medical And Biological Engineering

International Journal Of Case Reports And Therapeutic Studies | ISSN: 2689-310X

Journal Of Addiction \& Addictive Disorders | ISSN: 2578-7276

Journal Of Agronomy \& Agricultural Science | ISSN: 2689-8292

Journal Of AIDS Clinical Research \& STDs | ISSN: 2572-7370

Journal Of Alcoholism Drug Abuse \& Substance Dependence | ISSN: 2572-9594

Journal Of Allergy Disorders \& Therapy | ISSN: 2470-749X

Journal Of Alternative Complementary \& Integrative Medicine | ISSN: 2470-7562

Journal Of Alzheimers \& Neurodegenerative Diseases | ISSN: 2572-9608

Journal Of Anesthesia \& Clinical Care | ISSN: 2378-8879

Journal Of Angiology \& Vascular Surgery | ISSN: 2572-7397

Journal Of Animal Research \& Veterinary Science | ISSN: 2639-3751

Journal Of Aquaculture \& Fisheries | ISSN: 2576-5523

Journal Of Atmospheric \& Earth Sciences | ISSN: 2689-8780

Journal Of Biotech Research \& Biochemistry

Journal Of Brain \& Neuroscience Research

Journal Of Cancer Biology \& Treatment | ISSN: 2470-7546

Journal Of Cardiology Study \& Research | ISSN: 2640-768X

Journal Of Cell Biology \& Cell Metabolism | ISSN: 2381-1943

Journal Of Clinical Dermatology \& Therapy | ISSN: 2378-8771

Journal Of Clinical Immunology \& Immunotherapy | ISSN: 2378-8844

Journal Of Clinical Studies \& Medical Case Reports | ISSN: 2378-8801

Journal Of Community Medicine \& Public Health Care | ISSN: 2381-1978

Journal Of Cytology \& Tissue Biology | ISSN: 2378-9107

Journal Of Dairy Research \& Technology | ISSN: 2688-9315

Journal Of Dentistry Oral Health \& Cosmesis | ISSN: 2473-6783

Journal Of Diabetes \& Metabolic Disorders | ISSN: 2381-201X

Journal Of Emergency Medicine Trauma \& Surgical Care | ISSN: 2378-8798

Journal Of Environmental Science Current Research | ISSN: 2643-5020

Journal Of Food Science \& Nutrition | ISSN: 2470-1076

Journal Of Forensic Legal \& Investigative Sciences | ISSN: 2473-733X

Journal Of Gastroenterology \& Hepatology Research | ISSN: 2574-2566
Journal Of Genetics \& Genomic Sciences | ISSN: 2574-2485

Journal Of Gerontology \& Geriatric Medicine | ISSN: 2381-8662

Journal Of Hematology Blood Transfusion \& Disorders | ISSN: 2572-2999

Journal Of Hospice \& Palliative Medical Care

Journal Of Human Endocrinology | ISSN: 2572-9640

Journal Of Infectious \& Non Infectious Diseases | ISSN: 2381-8654

Journal Of Internal Medicine \& Primary Healthcare | ISSN: 2574-2493

Journal Of Light \& Laser Current Trends

Journal Of Medicine Study \& Research | ISSN: 2639-5657

Journal Of Modern Chemical Sciences

Journal Of Nanotechnology Nanomedicine \& Nanobiotechnology | ISSN: 2381-2044

Journal Of Neonatology \& Clinical Pediatrics | ISSN: 2378-878X

Journal Of Nephrology \& Renal Therapy | ISSN: 2473-7313

Journal Of Non Invasive Vascular Investigation | ISSN: 2572-7400

Journal Of Nuclear Medicine Radiology \& Radiation Therapy | ISSN: 2572-7419

Journal Of Obesity \& Weight Loss | ISSN: 2473-7372

Journal Of Ophthalmology \& Clinical Research | ISSN: 2378-8887

Journal Of Orthopedic Research \& Physiotherapy | ISSN: 2381-2052

Journal Of Otolaryngology Head \& Neck Surgery | ISSN: 2573-010X

Journal Of Pathology Clinical \& Medical Research

Journal Of Pharmacology Pharmaceutics \& Pharmacovigilance | ISSN: 2639-5649

Journal Of Physical Medicine Rehabilitation \& Disabilities | ISSN: 2381-8670

Journal Of Plant Science Current Research | ISSN: 2639-3743

Journal Of Practical \& Professional Nursing | ISSN: 2639-5681

Journal Of Protein Research \& Bioinformatics

Journal Of Psychiatry Depression \& Anxiety | ISSN: 2573-0150

Journal Of Pulmonary Medicine \& Respiratory Research | ISSN: 2573-0177

Journal Of Reproductive Medicine Gynaecology \& Obstetrics | ISSN: 2574-2574

Journal Of Stem Cells Research Development \& Therapy | ISSN: 2381-2060

Journal Of Surgery Current Trends \& Innovations | ISSN: 2578-7284

Journal Of Toxicology Current Research | ISSN: 2639-3735

Journal Of Translational Science And Research

Journal Of Vaccines Research \& Vaccination | ISSN: 2573-0193

Journal Of Virology \& Antivirals

Sports Medicine And Injury Care Journal | ISSN: 2689-8829

Trends In Anatomy \& Physiology | ISSN: 2640-7752

Submit Your Manuscript: https://www.heraldopenaccess.us/submit-manuscript 